\title{
OBTENÇÃO DAS ISOTERMAS DE SORÇÃO E MODELAGEM MATEMÁTICA PARA A PÊRA BARTLETT (Pyrus sp.) COM E SEM DESIDRATAÇÃO OSMÓTICA ${ }^{1}$
}

\author{
Kil Jin PARK ${ }^{2},{ }^{*}$, Adriana BIN ${ }^{3}$, \\ Fernando Pedro Reis BROD ${ }^{4}$
}

\begin{abstract}
RESUMO
Este trabalho aborda o estudo teórico-experimental da atividade de água de pêras in natura e desidratadas osmoticamente a 55Brix e $40^{\circ} \mathrm{C}$. As isotermas foram construídas para três níveis de temperatura $\left(40^{\circ} \mathrm{C}, 60^{\circ} \mathrm{C}\right.$ e $\left.80^{\circ} \mathrm{C}\right)$ e ajustadas pelos modelos mais usuais: Langmuir, BET, BET linear, GAB, Halsey, Oswin, Peleg, Chung e Henderson. O desvio relativo entre os valores experimentais e os valores estimados foi calculado para cada curva, a fim de se avaliar qual equação melhor se ajustou aos dados experimentais. O modelo que melhor tratou os resultados das isotermas de dessorção da pêra foi o de Henderson entre os modelos que englobam a temperatura. Em relação aos modelos que tratam as isotermas isoladamente, os modelos de Peleg, GAB e Oswin apresentaram o melhor ajuste para a pêra desidratada e in natura.
\end{abstract}

Palavras-chave: Curvas de equilíbrio; desidratação osmótica; modelos de isotermas.

\section{SUMMARY}

SORPTION ISOTHERMS DATA AND MATHEMATICAL MODELS FOR PEAR BARTLETT (Pyrus sp.) WITH AND WITHOUT OSMOTIC DEHYDRATION. This research work obtains the experimental data of pears in natura and osmotically dehydrated $\left(55^{\circ} \mathrm{Brix}\right.$ and $\left.40^{\circ} \mathrm{C}\right)$ for three different temperatures $\left(40^{\circ} \mathrm{C}, 60^{\circ} \mathrm{C}\right.$ and $80^{\circ} \mathrm{C}$ ). The desorption isotherms were fitted by the most usual models: Langmuir, BET, linear BET, GAB, Halsey, Oswin, Peleg, Chung and Henderson. The average relative deviations between experimental and calculated data were obtained to select the best model. The best model, with a temperature parameter, obtained for with and without osmotic dehydration, was the Henderson. In relation to the isotherms models, without temperature parameter, the best models were Peleg, GAB and Oswin.

Keywords: Equilibrium curves; osmotic dehydration; isotherms models.

\section{1 - INTRODUÇÃO}

A pêra é uma fruta original de zonas temperadas. A Pyrus communis L., cultivada na Europa é a mais importante das várias espécies existentes da fruta. $O$ sabor da pêra é associado com a quantidade de açúcares da mesma, que varia comumente de 8 a $11 \%$ de

\footnotetext{
${ }^{1}$ Recebido para publicação em 08/11/00. Aceito para publicação em 26/03/01.

2 Prof. Titular, Depto. Pré-Processamento de Produtos Agropecuários, Faculdade de Engenharia Agrícola, UNICAMP, Caixa Postal 6011, Campinas - SP, CEP 13081-970. Tel/Fax: (019) 3788-1076, e-mail:kil@agr.unicamp.br.

${ }^{3}$ Engenheira de Alimentos, FEA/UNICAMP, Campinas, SP.

${ }^{4}$ Doutorando em Engenharia Agrícola, FEAGRI/UNICAMP, Campinas, SP, e-mail:brod@agr.unicamp.br.

${ }^{*}$ A quem a correspondência deve ser enviada.
}

açúcar por unidade de peso de polpa. Além disso quanto mais suculenta a fruta, mais doce ela será.

$\mathrm{O} \mathrm{pH}$ das pêras varia de 2,6 a 5,4 , sendo encontrado principalmente o ácido málico e cítrico, além de outros ácidos orgânicos em menores quantidades. O sabor amargo está usualmente associado à casca, em função de substâncias fenólicas e polifenólicas. Os compostos voláteis aromáticos (77 componentes) contribuem para o sabor. A cor das cascas das pêras é dependente dos pigmentos das mesmas, principalmente a clorofila (verde) e carotenóide (amarelo). Já a polpa apresenta coloração próxima de creme [15].

As condições climáticas têm forte influência no armazenamento e na maturação das frutas. Para temperaturas muito altas ou muito baixas há uma redução no tamanho da fruta. Já o armazenamento a baixas temperaturas pode atenuar o sabor das frutas, já que ocorre um acúmulo de ácidos graxos, que é esterificado e volatilizado. Entretanto, há uma diminuição da taxa de respiração o que diminui a utilização de reservas das frutas e previne $o$ ataque de microorganismos.

A diminuição da atividade de água com a desidratação da fruta contribui para a conservação e conseqüente uso prolongado da fruta.

A desidratação osmótica é um processo que remove água dos alimentos sólidos. Como a atividade de água nos alimentos está relacionada com o crescimento de microorganismos e efetivação de reações químicas, a retirada de água reduz o crescimento destes microorganismos e impede reações bioquímicas que dependem da umidade, evitando a rápida perda que ocorre nos frutos in natura. Trata-se, portanto, de uma forma de conservação dos alimentos. Em seus estudos, ADAMBOUNOU, CASTAIGNE e DILLON [1], encontraram que o valor ótimo de atividade de água para o crescimento de microrganismos encontra-se entre 0,92 e 0,99. Além disso, a desidratação se revelou também como uma forma de reduzir os custos energéticos, importante para o transporte, embalagem e armazenamento dos alimentos com alto teor de água. Pode ser utilizada ainda, como pré-tratamento para alguns processos complementares já que encaminha duas transformações no alimento: a perda de água e a incorporação de certos solutos presentes no xarope.

Fruta cristalizada ou glaceada é o produto preparado com frutas, nas quais parte da água de sua composição é substituída por açúcares, através de uma tecnologia adequada. Estas frutas devem ser translúcidas, túrgidas, com consistência uniforme, isentas de granulosidades, com superfície seca e não áspera, cor 
e sabor agradáveis [10]. Neste experimento foi utilizado um método lento de açucaramento de pêras, através da imersão dos mesmos em xaropes de 40, 55 e $70^{\circ}$ Brix, com a constante circulação deste xarope através de uma bomba, como será descrito no item equipamento adiante.

A desidratação osmótica é uma eficiente forma de remoção de umidade dos alimentos sólidos, não ocorrendo mudança de fase da água [5]. Os alimentos, no caso as frutas, têm o seu peso reduzido para aproximadamente $50 \%$ do peso original pela desidratação osmótica. Este é um método utilizado para a obtenção de uma melhor qualidade das frutas e é utilizado como um estágio preliminar à secagem, pasteurização ou congelamento.

A secagem também provoca as mesmas alterações da desidratação osmótica, excluindo a incorporação de sólidos. Entretanto, a perda da quase totalidade da água livre presente não ocorre pela diferença de pressão osmótica, mas pela vaporização ou sublimação da água. Em secagens com ar quente esta vaporização ocorre pela diferença de temperatura entre ar quente e a fruta determinando uma diferença de pressão de vapor entre o ar e a superfície da fruta, e, ocasionando a transferência de massa de água para o ar, na forma de vapor de água.

A água é um dos mais importantes componentes dos alimentos, afetando todas as suas propriedades físicas. A forma como a água afeta a natureza física e as propriedades dos alimentos é complicada devido à interação entre a água e o meio, o que envolve a estrutura física, bem como a composição química dos diversos solutos incluindo polímeros e colóides ou partículas dispersas.

É possível estabelecer uma relação estreita entre o teor de água livre no alimento e sua conservação. 0 teor de água livre é expresso pela atividade de água $\left(\mathrm{a}_{\mathrm{w}}\right)$ que é dada pela relação entre a pressão de vapor de água em equilíbrio sobre o alimento, e a pressão de vapor de água pura, à mesma temperatura [12]. A atividade de água também pode ser entendida como a umidade relativa em equilíbrio com o produto na temperatura considerada.

O estudo da curva de sorção, que relaciona esta atividade de água com a umidade relativa de equilíbrio, fornece informações relevantes para adequação dos parâmetros de secagem. A determinação da atividade de água é uma das medidas mais importantes no processamento e na análise dos materiais biológicos, devido a sua importância no que diz respeito à qualidade e estabilidade. A afinidade existente entre a água e os outros componentes de um produto definem sua higroscopicidade que é muito marcante nos produtos e torna-se uma característica fundamental a influenciar os processos de manuseio, estocagem e consumo de materiais biológicos [18].

Quando um material biológico é exposto a uma certa umidade, ele perde ou ganha água para ajustar sua própria umidade a uma condição de equilíbrio com o ambiente. Isto ocorre quando a pressão de vapor d'água na superfície do material se iguala à pressão de vapor d'água do ar que o envolve [19].

As informações acerca da umidade de equilíbrio são importantes no processamento e armazenamento de alimentos . No processo de secagem, a umidade de equilíbrio é a umidade final, que o produto pode atingir se o processo for conduzido num longo período de tempo.

O estudo da atividade de água pode ser feito através das isotermas de sorção. Uma isoterma é uma curva que descreve, em uma umidade específica, a relação de equilíbrio de uma quantidade de água sorvida por componentes do material biológico e a pressão de vapor ou umidade relativa, a uma dada temperatura. Esta relação depende da composição química dos alimentos (gordura, amido, açúcar, proteínas, etc.).

Para determinação experimental de umidade de equilíbrio torna-se necessário um ambiente com umidade relativa controlada. $\mathrm{O}$ método de controle de umidade relativa mais usado é o que utiliza compostos químicos, tais como soluções aquosas de ácido sulfúrico e soluções de sais. Cada solução apresenta um grau de ajuste de umidade relativa que pode ser obtido variando-se a concentração das soluções a diferentes temperaturas.

Com o intuito de prever o comportamento das isotermas, diversos autores propuseram modelos de ajuste de isotermas de sorção. Estes modelos são úteis no conhecimento das características dos produto. Em sua maioria são modelos empíricos e são apresentados a seguir (Tabela 1).

TABELA 1. Modelos para ajustes de isotermas de sorção de umidade.

\begin{tabular}{ll}
\hline NOME DO MODELO & MODELO \\
\hline LANGMUIR [11] & $\frac{X_{\text {eq }}}{\mathrm{Xm}}=\frac{\mathrm{C} \cdot a_{\mathrm{w}}}{1+\mathrm{C} \cdot a_{\mathrm{w}}}$ \\
\hline $\begin{array}{l}\text { BET (BRUNAUER, EMMETT e } \\
\text { TELLER [6] }\end{array}$ & $X_{e q}=\frac{\left(X m \cdot C \cdot a_{w}\right) \cdot\left(1-(n+1) \cdot a_{w}{ }^{n}+n \cdot a_{w}{ }^{n+1}\right)}{\left(1-a_{w}\right) \cdot\left(1+(C-1) \cdot a_{w}-C \cdot a_{w}{ }^{n+1}\right)}$ \\
\hline $\begin{array}{l}\text { BET linearizado (BRUNAUER, } \\
\text { EMMET e TELLER [6]) }\end{array}$ & $\frac{a_{w}}{\left(1-a_{w}\right) \cdot X_{e q}}=\frac{1}{X m \cdot C}+\frac{a_{w} \cdot(C-1)}{X m \cdot C}$ \\
\hline $\begin{array}{l}\text { GAB - GUGGHENHEIM, } \\
\text { ANDERSON e de BOER (PARK } \\
\& \text { NOGUEIRA [13]) }\end{array}$ & $X_{e q}=\frac{X m \cdot C \cdot K \cdot a_{w}}{\left(1-K \cdot a_{w}\right) \cdot\left(1-K \cdot a_{w}+C \cdot K \cdot a_{w}\right)}$ \\
\hline HALSEY [9] & $a_{w}=\exp \left(\frac{-A}{X_{e q}{ }^{B}}\right)$ \\
\hline $\begin{array}{l}\text { OSWIN (CHINNAN \& } \\
\text { BEAUCHAT [7]) }\end{array}$ & $X_{e q}=A \cdot\left(\frac{a_{w}}{1-a_{w}}\right)^{B}$ \\
\hline PELEG [16] & $X_{e q}=k_{1} \cdot a_{w}{ }^{n_{1}}+k_{2} \cdot a_{w}{ }^{n_{2}}$ \\
\hline HENDERSON (ASAE [3]) & $1-A w=\exp \left[-\left(k \cdot X_{e q}{ }^{n}\right)\right]$ \\
\hline CHUNG \& PFOST [8] & Ln $a_{w}=-\frac{A}{R \cdot T} \cdot \exp \left[-B \cdot X_{e q}\right]$ \\
\hline
\end{tabular}

Onde:

$X_{\text {eq }}$ - conteúdo de umidade de equilíbrio, $\mathrm{kg} / \mathrm{kg}$;

$X_{m}$ - conteúdo de umidade na monocamada molecular, $\mathrm{kg} / \mathrm{kg}$;

$a_{w}$ - atividade de água, adimensional;

$\mathrm{T}$ - temperatura, ${ }^{\circ} \mathrm{C}$;

A, B, C, D, E, n, K, $\mathrm{k}_{1}, \mathrm{k}_{2}, \mathrm{n}_{1}, \mathrm{n}_{2}$ - constantes das equações.

$\mathrm{R}-$ constante universal de gases. 


\section{2 - MATERIAL E MÉTODOS}

A matéria-prima utilizada foi a pêra D'anjou, in natura e desidratada osmoticamente a 55B Brix e 40ํ $\mathrm{C}$ por 120 minutos. A fruta foi adquirida no comércio local e utilizada na forma de cubos de $1 \mathrm{~cm}$ de aresta.

Para a determinação da umidade de equilíbrio para pêra desidratada e pêra in natura foram utilizados sete sais em três diferentes temperaturas $\left(40^{\circ} \mathrm{C}, 60^{\circ} \mathrm{C}\right.$ e $\left.80^{\circ} \mathrm{C}\right)$. As amostras foram preparadas em triplicata. Na Tabela 2, encontram-se listados os sais utilizados e as umidades relativas correspondentes, nas temperaturas utilizadas.

TABELA 2. Atividade de água correspondente aos sais utilizados.

\begin{tabular}{cccc}
\hline \multirow{2}{*}{ SAIS } & \multicolumn{3}{c}{ Atividade de água (\%) } \\
\cline { 2 - 4 } $\mathrm{LiCl}$ & $\mathbf{4 0}^{\circ} \mathbf{C}$ & $\mathbf{6 0}^{\circ} \mathbf{C}$ & $\mathbf{8 0}^{\circ} \mathbf{C}$ \\
\hline $\mathrm{MgCl}_{2}$ & 11,21 & 10,95 & 10,51 \\
$\mathrm{NaBr}$ & 51,60 & 29,26 & 26,05 \\
$\mathrm{KI}$ & 66,09 & 49,66 & 51,43 \\
$\mathrm{NaNO}_{3}$ & 71,00 & 63,11 & 60,97 \\
$\mathrm{NaCl}$ & 74,68 & 67,35 & 65,22 \\
$\mathrm{KCl}$ & 82,32 & 74,50 & 76,29 \\
\hline
\end{tabular}

As pesagens periódicas das amostras foram feitas em uma balança analítica, até atingir peso constante, determinando-se assim, a umidade de equilíbrio. $O$ teor de umidade de equilíbrio (em base seca) será calculado, com base na diferença entre a massa da amostra no equilíbrio e a massa seca. A análise de regressão não linear do pacote estatístico STATISTICA [17] foi usada para estimar as constantes dos modelos de isotermas de dessorção para pêra in natura e desidratada.

Para confirmar que os parâmetros de regressão foram de fato únicos, a regressão foi repetida com estimativas de vários valores iniciais acima e abaixo dos calculados (método iterativo [16]). O critério usado para a boa avaliação foi o módulo do desvio relativo médio:

$$
E=\frac{100}{n e} \sum_{i=1}^{n e} \frac{|V E-V P|}{V E}
$$

Onde:

E - erro relativo médio; ne - número de dados experimentais;

VE - valor experimental;

VP - valor teórico.

Geralmente, são considerados que valores de desvio relativo médio abaixo de $10 \%$ indicam um razoável ajuste para as práticas propostas [2].

A massa seca da pêra foi determinada segundo a metodologia da $A O A C$ [4]: estufa à vácuo a $70^{\circ} \mathrm{C}$ por 24 horas.

\section{3 - RESULTADOS}

\section{1 - Isotermas de Dessorção}

A Tabela 3 relaciona os valores médios de umidade de equilíbrio com a atividade de água determinada pelos sais, para a construção das isotermas de dessorção.

TABELA 3. Umidade de equilíbrio para a pêra desidratada e in natura em função da temperatura e da atividade de água.

\begin{tabular}{|c|c|c|c|c|c|c|}
\hline & \multicolumn{2}{|c|}{$40^{\circ} \mathrm{C}$} & \multicolumn{2}{|c|}{$60^{\circ} \mathrm{C}$} & \multicolumn{2}{|c|}{$80^{\circ} \mathrm{C}$} \\
\hline & $\mathbf{a}_{w}$ & $\mathbf{X}_{\mathrm{eq}}$ & $\mathbf{a}_{w}$ & $\mathbf{X}_{\mathrm{eq}}$ & $\mathbf{a}_{w}$ & $\mathbf{X}_{\mathrm{eq}}$ \\
\hline \multirow{7}{*}{ 焉 } & 0,1121 & 0,0652 & 0,1095 & 0,0357 & 0,1051 & 0,0042 \\
\hline & 0,3160 & 0,1035 & 0,2926 & 0,0740 & 0,2605 & 0,0205 \\
\hline & 0,5317 & 0,1483 & 0,4966 & 0,0744 & 0,5143 & 0,0654 \\
\hline & 0,6609 & 0,2151 & 0,6311 & 0,1161 & 0,6097 & 0,0744 \\
\hline & 0,7468 & 0,5167 & 0,6735 & 0,1224 & 0,6522 & 0,0751 \\
\hline & 0,8021 & 0,7202 & 0,7450 & 0,3527 & 0,7629 & 0,1886 \\
\hline & & & 0,8025 & 0,5625 & 0,7890 & 0,1941 \\
\hline \multirow{7}{*}{ 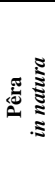 } & 0,1121 & 0,0736 & 0,1095 & 0,0647 & 0,1051 & 0,0075 \\
\hline & 0,3160 & 0,0899 & 0,2926 & 0,0675 & 0,2605 & 0,0093 \\
\hline & 0,5317 & 0,2045 & 0,4966 & 0,1152 & 0,5143 & 0,0456 \\
\hline & 0,6609 & 0,2622 & 0,6311 & 0,2137 & 0,6097 & 0,0336 \\
\hline & 0,7468 & 0,4477 & 0,6735 & 0,2399 & 0,6522 & 0,0907 \\
\hline & 0,8232 & 0,7290 & 0,7450 & 0,4099 & 0,7629 & 0,1895 \\
\hline & & & 0,8025 & 0,6028 & 0,7890 & 0,2073 \\
\hline
\end{tabular}

\section{2 - Modelos matemáticos}

As isotermas de dessorção foram ajustadas para os modelos descritos. Nas Tabelas 4 e 5 são mostrados os valores dos parâmetros calculados para os ajustes, além do coeficiente de determinação $\left(R^{2}\right)$ e do erro relativo médio $(E)$.

TABELA 4. Valores dos parâmetros dos modelos de isotermas da pêra desidratada.

\begin{tabular}{|c|c|c|c|c|}
\hline \multirow{2}{*}{ MODELO } & \multirow{2}{*}{ CONSTANTES } & \multicolumn{3}{|c|}{ TEMPERATURA } \\
\hline & & $40^{\circ} \mathrm{C}$ & $60^{\circ} \mathrm{C}$ & $80^{\circ} \mathrm{C}$ \\
\hline \multirow{5}{*}{ BET } & $\mathrm{XM}$ & 0,295 & 34,384 & 0,062 \\
\hline & $\mathrm{C}$ & 0,242 & 0,001 & 0,624 \\
\hline & $\mathrm{N}$ & 27,082 & 29,276 & 26,048 \\
\hline & $\mathrm{R}^{2}$ & 0,98 & 0,98 & 0,99 \\
\hline & $\mathrm{E}(\%)$ & 31,09 & 36,98 & 15,10 \\
\hline \multirow{4}{*}{ BET Linear } & XM & 0,277 & 16,129 & 0,060 \\
\hline & $\mathrm{C}$ & 0,266 & 0,002 & 0,660 \\
\hline & $\mathrm{R}^{2}$ & 0,98 & 0,98 & 0,99 \\
\hline & $\mathrm{E}(\%)$ & 31,40 & 37,01 & 14,91 \\
\hline \multirow{5}{*}{ GAB } & XM & 0,072 & 0,039 & 0,035 \\
\hline & $\mathrm{C}$ & 13,276 & 20,255 & 1,764 \\
\hline & $\mathrm{K}$ & 1,125 & 1,162 & 1,071 \\
\hline & $\mathrm{R}^{2}$ & 0,99 & 0,98 & 0,99 \\
\hline & $\mathrm{E}(\%)$ & 24,39 & 20,13 & 19,33 \\
\hline \multirow{4}{*}{ HALSEY } & A & 0,178 & 0,166 & 0,072 \\
\hline & B & 0,666 & 0,499 & 0,747 \\
\hline & $\mathrm{R}^{2}$ & 0,99 & 0,98 & 0,99 \\
\hline & $\mathrm{E}(\%)$ & 49,49 & 35,82 & 28,57 \\
\hline \multirow{4}{*}{ LAGMUIR } & XM & 1015,778 & 3804,041 & 807,970 \\
\hline & $\mathrm{C}$ & $5,93 \times 10^{-4}$ & $1,02 \times 10^{-4}$ & $2,27 \times 10^{-4}$ \\
\hline & $\mathrm{R}^{2}$ & 0,79 & 0,69 & 0,84 \\
\hline & $\mathrm{E}(\%)$ & 55,70 & 74,45 & 99,09 \\
\hline \multirow{4}{*}{ OSWIN } & A & 0,120 & 0,049 & 0,048 \\
\hline & B & 1,282 & 1,746 & 1,091 \\
\hline & $\mathrm{R}^{2}$ & 0,98 & 0,98 & 0,98 \\
\hline & $\mathrm{E}(\%)$ & 31,19 & 39,72 & 14,40 \\
\hline \multirow{6}{*}{ PELEG } & $\mathrm{k}_{1}$ & 0,129 & 0,071 & 0,115 \\
\hline & $\mathrm{n}_{1}$ & 0,275 & 0,201 & 1,220 \\
\hline & $\mathrm{k}_{2}$ & 3,231 & 4,039 & 0,965 \\
\hline & $\mathrm{n}_{2}$ & 7,537 & 9,458 & 8,853 \\
\hline & $\mathrm{R}^{2}$ & 0,99 & 0,99 & 0,99 \\
\hline & $\mathrm{E}(\%)$ & 9,14 & 15,34 & 19,51 \\
\hline \multirow{5}{*}{ CHUNG \& PFOST } & $\mathrm{E}$ & & 0,730 & \\
\hline & D & & 0,176 & \\
\hline & $\mathrm{C}$ & & 20,546 & \\
\hline & $\mathrm{R}^{2}$ & & 0,82 & \\
\hline & $\mathrm{E}(\%)$ & & 239,50 & \\
\hline \multirow{5}{*}{ HENDERSON } & $\mathrm{K}$ & & 0,019 & \\
\hline & $\mathrm{C}$ & & 55,417 & \\
\hline & $\mathrm{N}$ & & 0,385 & \\
\hline & $\mathrm{R}^{2}$ & & 0,97 & \\
\hline & $\mathrm{E}(\%)$ & & 43,28 & \\
\hline
\end{tabular}


TABELA 5. Valores dos parâmetros dos modelos de isotermas da pêra in natura

\begin{tabular}{|c|c|c|c|c|}
\hline \multirow{2}{*}{ MODELO } & \multirow{2}{*}{ CONSTANTES } & \multicolumn{3}{|c|}{ TEMPERATURA } \\
\hline & & $40^{\circ} \mathrm{C}$ & $60^{\circ} \mathrm{C}$ & $80^{\circ} \mathrm{C}$ \\
\hline \multirow{5}{*}{ BET } & $\mathrm{XM}$ & 0,148 & 0,185 & 2,024 \\
\hline & $\mathrm{C}$ & 1,216 & 0,433 & 0,008 \\
\hline & $\mathrm{N}$ & 175,535 & 144,687 & 12,023 \\
\hline & $\mathrm{R}^{2}$ & 0,99 & 0,99 & 0,99 \\
\hline & $\mathrm{E}(\%)$ & 19,19 & 20,43 & 29,22 \\
\hline \multirow{4}{*}{ BET Linear } & XM & 0,148 & 0,185 & 0,130 \\
\hline & $\mathrm{C}$ & 1,216 & 0,433 & 0,145 \\
\hline & $\mathrm{R}^{2}$ & 0,99 & 0,99 & 0,98 \\
\hline & $\mathrm{E}(\%)$ & 19,19 & 20,43 & 31,59 \\
\hline \multirow{5}{*}{ GAB } & XM & 0,086 & 0,065 & 1,743 \\
\hline & $\mathrm{C}$ & 12,376 & 12,509 & 0,011 \\
\hline & $\mathrm{K}$ & 1,073 & 1,115 & 0,943 \\
\hline & $\mathrm{R}^{2}$ & 0,99 & 0,99 & 0,98 \\
\hline & $\mathrm{E}(\%)$ & 10,67 & 12,13 & 29,38 \\
\hline \multirow{4}{*}{ HALSEY } & A & 0,147 & 0,153 & 0,098 \\
\hline & B & 0,853 & 0,717 & 0,580 \\
\hline & $\mathrm{R}^{2}$ & 0,99 & 0,99 & 0,98 \\
\hline & $\mathrm{E}(\%)$ & 11,42 & 14,23 & 26,52 \\
\hline \multirow{4}{*}{ LAGMUIR } & XM & 1596,919 & 488,629 & 869,934 \\
\hline & $\mathrm{C}$ & $3,80 \times 10^{-4}$ & $1,00 \times 10^{-3}$ & $2,03 \times 10^{-4}$ \\
\hline & $\mathrm{R}^{2}$ & 0,83 & 0,80 & 0,77 \\
\hline & $\mathrm{E}(\%)$ & 43,96 & 52,45 & 135,87 \\
\hline \multirow{4}{*}{ OSWIN } & A & 0,158 & 0,114 & 0,032 \\
\hline & B & 0,981 & 1,177 & 1,443 \\
\hline & $\mathrm{R}^{2}$ & 0,99 & 0,99 & 0,98 \\
\hline & $\mathrm{E}(\%)$ & 20,28 & 20,34 & 33,32 \\
\hline \multirow{6}{*}{ PELEG } & $\mathrm{k}_{1}$ & 0,243 & 0,103 & 0,026 \\
\hline & $\mathrm{n}_{1}$ & 0,641 & 0,241 & 0,585 \\
\hline & $\mathrm{k}_{2}$ & 2,372 & 2,090 & 0,821 \\
\hline & $\mathrm{n}_{2}$ & 7,845 & 6,463 & 6,120 \\
\hline & $\mathrm{R}^{2}$ & 0,99 & 1,00 & 0,99 \\
\hline & $\mathrm{E}(\%)$ & 11,43 & 5,72 & 23,42 \\
\hline \multirow{5}{*}{ CHUNG \& PFOST } & $\mathrm{E}$ & & 0,797 & \\
\hline & D & & 0,187 & \\
\hline & $\mathrm{C}$ & & 17,428 & \\
\hline & $\mathrm{R}^{2}$ & & 0,85 & \\
\hline & $\mathrm{E}(\%)$ & & 175,02 & \\
\hline \multirow{5}{*}{ HENDERSON } & $\mathrm{K}$ & & 0,023 & \\
\hline & $\mathrm{C}$ & & 47,181 & \\
\hline & $\mathrm{N}$ & & 0,526 & \\
\hline & $\mathrm{R}^{2}$ & & 0,95 & \\
\hline & $\mathrm{E}(\%)$ & & 50,35 & \\
\hline
\end{tabular}

\section{3 - Umidade de Equilíbrio}

Ajustando as isotermas de dessorção da pêra desidratada e in natura para os modelos matemáticos mais usuais, obtivemos $R^{2}$ e o erro relativo (\%) para cada um destes modelos. A escolha do modelo mais adequado para o cálculo da umidade de equilíbrio baseou-se no valor do erro relativo.

Segundo esta análise determinou-se o modelo de Henderson (dentre os modelos com o parâmetro temperatura) e o modelo de Peleg, Oswin e GAB (dentre os modelos sem o parâmetro temperatura) como os que apresentaram melhores ajustes às isotermas, tanto para pêra in natura quanto para a pêra desidratada.

As comparações entre valores experimentais e previstos para as temperaturas de 40,60 e $80^{\circ} \mathrm{C}$, estão descritos nas Figuras 1 a 3.

PARK, YADO e BROD [14] determinaram as isotermas de sorção para a pêra in natura nas temperaturas de 40,60 e $70^{\circ} \mathrm{C}$. O modelo que apresentou 0 melhor ajuste também foi o de Peleg. A Figura $4 \mathrm{com}$ para o presente trabalho com o anterior para a temperatura de $60^{\circ} \mathrm{C}$, mostrando uma coerência dos resultados, apesar da matéria-prima ter sido obtida em épocas diferentes.

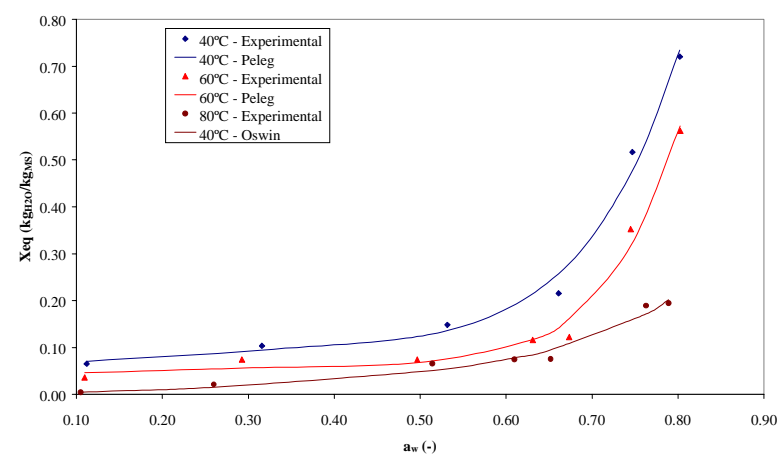

FIGURA 1. Comparação entre os valores observados e predidos para a Isoterma de Dessorção da Pêra Desidratada para os modelos sem o parâmetro temperatura.

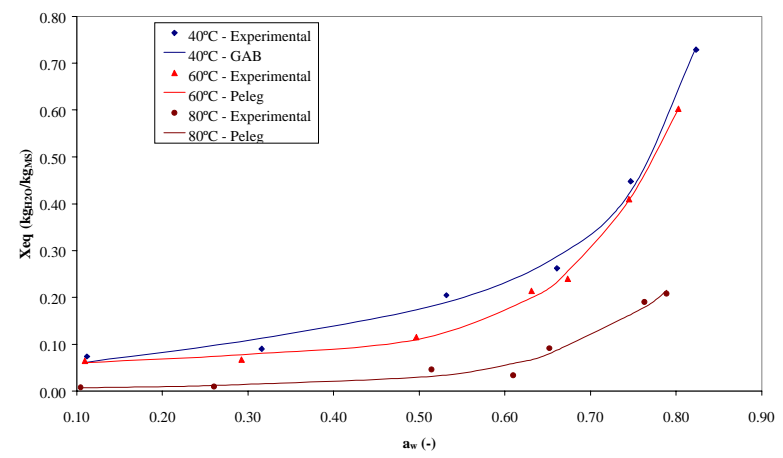

FIGURA 2. Comparação entre os valores observados e predidos para a Isoterma de Dessorção da Pêra in natura para os modelos sem o parâmetro temperatura.

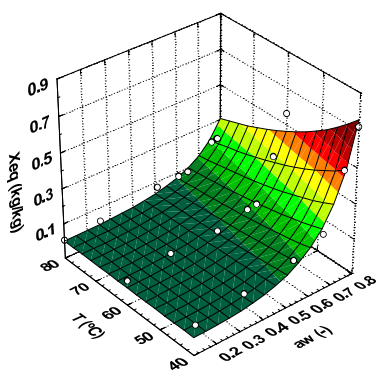

Pêra desidratada Modelo de Henderson

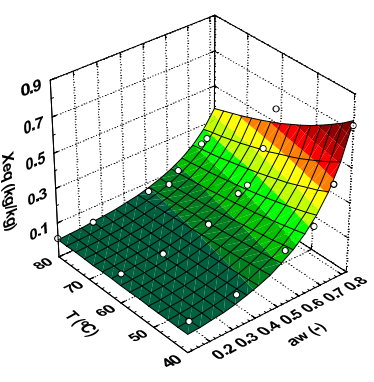

Pêra in natura Modelo de Henderson
FIGURA 3. Comparação entre os valores observados e predidos para a Isoterma de Dessorção da Pêra Desidratada e in natura para o modelo de Henderson com o parâmetro temperatura.

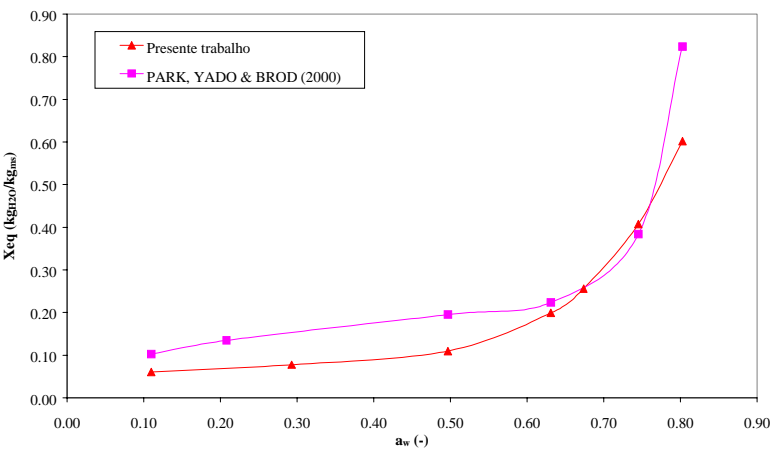

FIGURA 4. Comparação entre as isotermas de sorção de $60^{\circ} \mathrm{C}$ para a pêra in natura. 


\section{4 - CONCLUSÕES}

- As isotermas de dessorção para pêra sem tratamento (in natura) e tratadas osmoticamente determinadas para três temperaturas diferentes ( 40 , 60 e $80^{\circ} \mathrm{C}$ ), foram ajustadas com os modelos sem o parâmetro temperatura (BET, BET linear, GAB, Halsey, Lagmuir, Oswin e Peleg) e os com o parâmetro temperatura (Henderson e Chung \& Pfost).

- A avaliação do melhor ajuste foi feita pelo valor do desvio relativo entre os valores experimentais e os valores estimados e o modelo que meIhor representou os resultados das isotermas de dessorção da pêra, foi o de Henderson entre os modelos com o parâmetro temperatura, apresentando os valores de erro relativo de $43,28 \%$ para a pêra desidratada e de $50,35 \%$ para a pêra in natura.

- Em relação aos modelos sem o parâmetro temperatura, o modelo de Peleg apresentou o meIhor ajuste para a pêra desidratada para a temperatura de $40^{\circ} \mathrm{C}(9,14 \%)$ e $60^{\circ} \mathrm{C}(15,34 \%)$, enquanto que para a temperatura de $80^{\circ} \mathrm{C}$ o modelo de Oswin apresentou o menor erro relativo $(14,40 \%)$. Para a pêra in natura o modelo de $G A B$ foi o melhor ajuste para a temperatura de $40^{\circ} \mathrm{C}$ $(10,67 \%)$ e para as temperaturas de $60^{\circ} \mathrm{C}$ $(5,72 \%)$ e de $80^{\circ} \mathrm{C}(23,42 \%)$ o modelo de Peleg foi o melhor ajuste.

- Para as três temperaturas, na região intermediária de atividade de água, as amostras tratadas osmoticamente exibiram uma clara tendência de apresentar conteúdos de umidade de equilíbrio menores do que as amostras in natura.

\section{5 - REFERÊNCIAS BIBLIOGRÁFICAS}

[1] ADAMBOUNOU, T.L., CASTAIGNE, F., DILLON, J.C. Abaissement de l'activité de l'eau de légumes tropicaux par déshydration osmotique partielle. Sciences des Aliments, Paris: Lavoisier Abonnements, v.3, n. 4, p. 551567, 1983.

[2] AGUERRE, R.J., SUAREZ, C., VIOLLAZ, P.E. New BET type multilayer sorption isotherms. Part II: Modeling water sorption in foods, Lebensmittel-Wissenschaft \& Technologie, London: Academic Press Ltd., v.22, n.4, p.192-195, 1989.

[3] AMERICAN SOCIETY OF AGRICULTURAL ENGINEERS - ASAE. Moisture Relationship of Grains. St. Joseph, 1991 b, p.363-7. (ASAE Data, D245.4).

[4] ASSOCIATION OF OFFICIAL ANALYTICAL CHEMISTS AOAC. Official Methods of Analysis: 930.04, Moisture Content in Plants, 1990, v. 1.
[5] BOLIN, H.R; HUXSOLL, C.C. Partial Drying of Cut Pears to Improve Freeze/Thaw Texture. Journal of Food Science, Chicago: Inst Food Technologists, v. 58, n. 2, p. 357-360, 1993.

[6] BRUNAUER, S., EMMET, T.H., TELLER, F. Adsorption of gases in multmolecular layers. J. Am. Chem. Soc., Easton, PA: Mack Printing Company, v. 60, n. 2, p. 309-319, 1938.

[7] CHINNAN, M.S., BEAUCHAT, L.R. Sorption isotherms of whole cowpeas and flours. Lebensmittel-Wissenschaft \& Technologie, London: Academic Press Ltd., v. 18, p. 8388, 1985.

[8] CHUNG, D.S., PFOST, H.B. Transactions of the ASAE, St Joseph: Amer. Soc. Agricultural Engineers, v.10, p.594, 1967.

[9] HALSEY, G. Physical adsorption on uniform surfaces. Journal of Chemical Physics, Woodbury: Amer. Inst. Physics, v. 16, n. 10, p. 931-937, 1985.

[10] JACKIX, M. H. Industrialização de Frutas em Calda e Cristalizadas, Geléias e Doces em Massa. 1982, [s.I.]: Fundação Tropical de Pesquisas e Tecnologia.

[11] LANGMUIR, I. The adsorption of gases in a plane surfaces of glass, mica and platinum.J. Am. Chem. Soc., v. 46, p. 1361-1403, 1918.

[12] MOHSENIN, N.N. Physical properties of plants and animals materials. 2. ed. Amsterdam: Gordon and Breach Publishers, 1986, $841 \mathrm{p}$.

[13] PARK, K.J., NOGUEIRA, R.I. Modelos de ajuste de isotermas de sorção de alimentos. Engenharia Rural, Piracicaba: ESALQ/USP, v. 3, n. 1, p.81-86, 1992.

[14] PARK, K.J., YADO, M.K.M., BROD, F.P.R. Isotermas de dessorção para a pêra bartlett (Pyrus sp.). Engenharia Rural. Piracicaba: ESALQ/USP, 2000 (submetido).

[15] PATTEE, H.E. Evaluation of quality of fruits and vegetables, Westport: Avi publishing company linc., 1985, p. 47-59.

[16] PELEG, M. Assessment of a semi-empirical four parameter general model for sigmoid moisture sorption isotherms. Journal of Food Processing Engineering, ConnecticutUSA: Food \& Nutrition Press Inc., v. 16, n. 1, p. 21-37, 1993.

[17] STATISTICA for Windows 5.0. Computer program manual. Tulsa, OK: StatSoft, Inc., 1995.

[18] TEXEIRA NETO, R. O., QUAST, D. G. Isotermas de adsorção de umidade em alimentos. Campinas: ITAL, v. 8, p. 141-197, 1993.

[19] TREYBALL, R.B. Drying. Mass Tranfer Operations. New York: McGraw-Hill, 1968, p. 569-575.

\section{6 - AGRADECIMENTOS}

Agrademos à Fundação de Amparo à Pesquisa do Estado de São Paulo - FAPESP, pela concessão da bolsa de Iniciação Científica. 